\title{
Kernos
}

Revue internationale et pluridisciplinaire de religion grecque antique

15 | 2002

Varia

\section{La « rationalité » des mythes de Delphes : les} dieux, les héros, les médiateurs

\section{Emilio Suárez de la Torre}

\section{(2) OpenEdition}

\section{Journals}

Édition électronique

URL : http://journals.openedition.org/kernos/1374

DOI : 10.4000/kernos. 1374

ISSN : 2034-7871

Éditeur

Centre international d'étude de la religion grecque antique

Édition imprimée

Date de publication : 1 janvier 2002

ISSN : 0776-3824

Référence électronique

Emilio Suárez de la Torre, «La « rationalité » des mythes de Delphes : les dieux, les héros, les médiateurs », Kernos [En ligne], 15 | 2002, mis en ligne le 21 avril 2011, consulté le 20 avril 2019. URL http://journals.openedition.org/kernos/1374; DOI : 10.4000/kernos.1374 


\section{La « rationalité 》 des mythes de Delphes ${ }^{1}$ : les dieux, les héros, les médiateurs}

\section{Introduction}

Le but de cette analyse est de montrer que les mythes qui sont nés autour du sanctuaire delphique forment un système auquel préside une remarquable rationalité. Mon point de départ est la conviction que l'opposition radicale entre mythos et logos, telle qu'elle a été formulée parfois au cours du $\mathrm{xx}^{\mathrm{e}}$ siècle, doit être nuancée ${ }^{2}$. Je me permets de citer à cet égard Hans Georg Gadamer : "Il suffit de lire Homère pour reconnaître la séduisante rationalité avec laquelle la mythologie grecque interprète l'existence humaine ${ }^{3}$. Le logos n'a plus même le monopole de la démonstration et de l'explication.

D'un côté, Héraclite nous a appris que le dieu de Delphes oüte $\lambda \epsilon ́ \gamma \epsilon l$

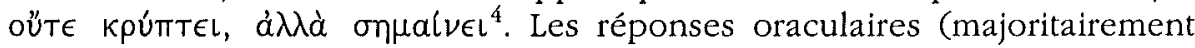
transmises dans les sources littéraires) possèdent une inquiétante ambiguilté. D'autre part, Delphes devient le centre de la prescription sans équivoque, autant dans le cas des réponses prostagmatiques qu'au moment de se constituer en dépôt ou noyau de la sagesse qui règle la conduite des hommes:

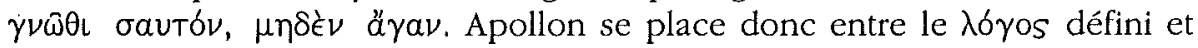

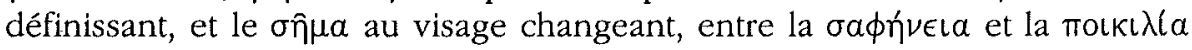
sémantique. Mais, entre ces deux extrêmes se trouve aussi la complexité du $\mu \hat{u} \theta 0 s$. Delphes est le domaine de la parole et de l'image. Elles composent un tout. Non seulement l'image offerte par le paysage (le domaine de la nature), mais aussi celle qui a été créée par l'homme. Des centaines d'objets divers, des statues surtout, mais aussi des peintures, pour ne pas parler des inscriptions, renferment des récits potentiels qui se déploient au moment d'être contemplés par les visiteurs : des héros, des personnages historiques plus ou moins proches ou lointains, des animaux qui faisaient surgir les légendes et les événements les plus extraordinaires (le cheval, le bison, la

1 Étude réalisée dans le cadre du Programme de Recherche PB 97-0403 de la DGICYT espagnole. Je remercie mon grand ami et collègue André Motte pour sa révision d'une première version française, ainsi que pour ses indications concernant le contenu.

2 Je renvoie au volume collectif édité par Buxton (1999).

3 Extrait de Mythos und Vernunft, in Gesammelte Werke VIII, Ästhetik und Poetik I, Kunst als Aussage, Tübingen, 1993, p. 163-169 (p. 169).

4 HÉrACL. B 93 D-K. 
chèvre, le loup, l'âne). Toutes choses auxquelles s'ajoutent les thèmes des chants dans les célébrations, les récits de la lescbé, etc.

Étant donné la variété et l'abondance des mythes à traiter, je vais seulement choisir (et présenter d'une façon parfois trop synthétique) ceux qui sont nés autour des dieux et des mortels, en laissant à une étude plus large la totalité de la mythologie delphique, qui devraient embrasser également des mythes sur les éléments non humains ${ }^{5}$.

\section{Dieux et déesses}

\subsection{Cronos et Zeus}

Un mythe soulignait l'appartenance de Delphes au «nouvel ordre » de Zeus. La pierre qui avait été avalée par Cronos, comme conséquence de la ruse de Gè, et qu'il vomit avec les autres dieux, avait été déposée à Delphes par Zeus ${ }^{6}$, d'après Hésiode, afin qu'elle fût un ô̂ $\mu \alpha$ pour les générations futures. Chaque jour on y versait de l'huile et les jours de fête on la couvrait de laine vierge ${ }^{7}$.

\subsection{Apollon}

\subsubsection{L'occupation du territoire et la consolidation du pouvoir du dieu}

Le corps principal des mythes delphiques avait pour but de justifier la présence apollinienne dans le sanctuaire et de nous présenter Apollon comme un Olympien vainqueur des forces chthoniennes anciennes. Il s'agit d'un constructum qui a égaré longtemps même les archéologues, au moment d'identifier les vestiges de la phase apparemment ancienne ${ }^{8}$. La plupart des récits décrivent une occupation par la force, après la victoire du dieu sur plusieurs ennemis et quelques difficultés. En réalité, il s'agit d'une sorte de mythe de fondation. Le dieu qui préside aux voyages de colonisation est lui-

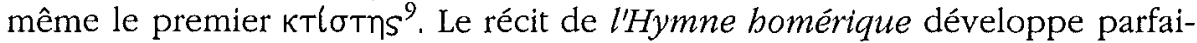
tement cette idée. Même dans le fait que le dieu est présenté en principe comme une menace pour la stabilité du panthéon divin : l'élan et la force du jeune dieu est 'reconduite' et transformée en procès fondateur et civilisateur,

5 Le texte présenté à l'occasion du Colloque de Rhodes contenait des remarques sur les mythes tendant à expliquer les éléments non humains de l'ensemble delphique : le paysage et les temples. Le développement de tous les points aurait exigé un nombre de pages excédant des limites prévues. J'ai donc concentré mon analyse sur le groupe des dieux, héros et hommes, ce qui permet en même temps une plus grande cohérence.

6 Cf. HÉs., Th., 498-500.

7 Paus., X, 24, 6. Le Périégète dit que cette histoire est une $\delta 6 \xi \alpha$. En effet, tout indique qu'il s'agit d'un rituel très ancien (peut être un reste d'une période de culte aniconique) interprété dans le cadre de l'idéologie « théogonique ».

$8 C f$. ce qui se passe avec Dionysos, présenté comme un « nouveau venu » rejeté et pour lequel on proposait une arrivée secondaire en Grèce, de Thrace ou de Phrygie. Mais les tablettes mycéniennes ont rendu ces hypothèses caduques.

9 Cf. Detienne (1997), Malkin (2000). 
après avoir surmonté des épreuves qui confirment ses pouvoirs. Le modèle choisi pour décrire la consolidation de ces pouvoirs est bien attesté dans de nombreuses cultures : c'est le combat myth, largement analysé par Fontenrose $^{10}$ et révisé récemment par Watkins dans le cadre de la mythologie et des traditions poétiques indoeuropéennes ${ }^{11}$. Par contre, au v $\mathrm{v}^{\mathrm{e}}$ siècle, le théâtre athénien se fait écho d'une version de l'occupation qui n'est pas forcement violente : les divinités féminines anciennes « cèdent » la place au jeune dieu.

\subsection{1. Épuration du territoire. Occupation violente}

\subsubsection{La menace extérieure, les monstres et les brigands ${ }^{12}$}

Ces êtres ont tous en commun d'appartenir aux territoires voisins ou d'être liés à l'histoire du sanctuaire (e.g. par l'existence d'une "voie sacrée "). Quelques-uns reflètent une violence, disons, «personnelle », contre le dieu ou les déesses de sa famille (c'est le cas de Tityos) ou bien ils sont des brigands qui attaquent les gens en chemin vers Delphes (Phorbas, Cycnos, Pyréneus, Sybaris, etc.). D'autres nous parlent d'une hostilité collective: ce sont les peuples dont on disait qui avaient envahi ou attaqué jadis le territoire de la Phocide et dont on soulignait l'impiété et l'bybris. Tous ces mythes ne sont pas, à proprement parler, « apolliniens », mais parfois « héracléens ». Pourtant, les récits montrent la « collaboration » apportée au dieu par le héros thébain, qui souvent lui fait des offrandes après ses victoires ${ }^{13}$.

\subsubsection{Python et le "mythe de combat ${ }^{14}$. Son rapport avec Typhon ${ }^{15}$}

Le parallèle avec le mythe de Typhon est indéniable ${ }^{16}$. Le plus important est que, de la même façon que le combat de Zeus contre Typhon sert à renforcer la légitimation du pouvoir acquis par le nouveau souverain, la victoire sur la dragonne confirme le droit d'Apollon sur le lieu choisi. En outre, c'est le grand mythème du sanctuaire, car il sert d'étiologie à plus d'une fête locale : d'un côté, pour une partie du rituel du septérion, d'un autre pour les Jeux Pythiques (interprétés officiellement comme une institution destinée à commémorer la victoire du dieu ou comme rite funéraire).

L'Hymne bomérique à Apollon, dans sa partie « delphique », est un exemple très important de cristallisation du mythe de la fondation du sanc-

10 Fontenrose (1959).

11 Watkins (1995), p. 460-463 pour Python (le livre propose une riche étude de la présence du motif - et des formules poétiques employées - dans plusieurs traditions).

12 Tityos, Phorbas, Phlégyas (et les Phlégyens), Cycnos; les Lapithes, les Dryopes et d'autres: Coronos, Laogoras, Erginos, Amyntor, Eurytos, Pyréneus, Sybaris.

13 Cf. Fontenrose (1959), 35 sq.

14 Cf. RuthERFord (2001), p. 397.

15 Pour la «typologie " du monstre, $c f$. les remarques de Fontenrose (1959) sur d'autres monstres féminins : Échidna et Cétos; Scylla, Lamie, Poiné et Psamathé; Empousa, Mormo, Gelo.

16 Cf. également Fontenrose (1959), p. 77 sq. 
tuaire par le dieu. Non seulement le dieu accomplit sa fonction de fondateur, mais aussi une longue série d'éléments du récit sont en rapport très direct avec les institutions, l'histoire et les fondements idéologiques de la religion delphique. Le parcours du dieu, ses victoires sur plusieurs monstres (et, surtout, la dragonne), l'organisation du premier pèlerinage ${ }^{17}$, où le dieu est accompagné des orgêones, la fondation du culte oraculaire (et d'autres manifestations du culte apollinien, comme celle d'Apollon Delphinios) et les procédés de sacrifice, consultation, etc., tout cela donne au texte une valeur étiologique tout à fait spéciale. Je ne suivrai pas Kolk ${ }^{18}$, lorsqu'il propose de voir dans cet hymne une composition faite exprès pour le rituel du septérion ou la tradition du nomos pythique, mais il faut reconnaître la valeur que ce texte possède en raison des nombreuses réalités historiques qu'il reflète. Même l'importance de la composante crétoise pourrait être expliqué, non seulement par l'antiquité réelle des traditions apolliniennes de l'île (purification, « origines » du péan, etc.), mais aussi (et surtout) par la forte influence de cette île dans les traditions religieuses de la Grèce pendant la période archaïque (en tout cas, il ne faut pas oublier l'importance des rapports du sanctuaire avec la Crète pendant cette même période).

Les mythes relatifs au phénomène de la purification ont leur raison d'être et dans la nature même de la religion apollinienne et dans les procédés de consultation des oracles. D'un côté, l'état de pureté est indispensable pour poser la question au dieu, mais, d'un autre (encore plus important) c'est un concept fondamental de la morale delphique (très enraciné d'ailleurs dans la religion grecque), mis très tôt en rapport avec la souillure provoquée par les crimes de sang. Le dieu même devient un paradigme de cette mentalité dans la version qui explique pourquoi le rituel du septérion a comme contexte la vallée de Tempè (en Thessalie), en tant qu'évocation du voyage apollinien.

Les descriptions plus détaillées se trouvent chez Plutarque ${ }^{19}$ et Élien ${ }^{20}$, mais nous pouvons à présent confirmer l'antiquité du rituel. Les plus récentes interprétations d'un fragment de papyrus pindarique (appartenant à un péan $^{21}$ ) montrent que le Thébain connaissait le rite du septérion et les mythes qui l'expliquaient. Tout devient extrêmement cohérent. Le dieu a tué un monstre chthonien et il part pour un territoire où, d'après Homère ${ }^{22}$, un fleuve (le Titarésios) entre en contact (sans se mêler) avec un autre (le Pénéios) qui provient des profondeurs de la terre (car il naît du Styx). Il établit de cette façon un modèle de voyage initiatique, un lien entre deux centres

\footnotetext{
17 Cf. MOTTE (1992).

18 Kolk (1963).

19 De defectu oraculorum, $15(418 \mathrm{a}-\mathrm{b})$

20 V.H. III,1.

21 C'est le Péan X (a) Maehler (=A2 Rutherford). Cf. Suárez de la Torre (1998c) et Rutherford (2001), p. 200-207. Les scholies permettent de se faire une idée du contenu.

22 Il. II, 755.
} 
apolliniens (et avec la Thessalie, l'État le plus important pendant la phase ancienne de l'Amphictionie) ${ }^{23}$ et aussi, en revenant avec le laurier, un lien entre les deux rituels principaux de Delphes. Ces deux rites, les Jeux Pythiques et le Septérion, ont partagé la même périodicité (huit ans) et ont peutêtre été célébrés pendant la même année dans une phase archaïque.

En outre, la perspective chthonienne proposée par Rutherford ${ }^{24}$ comme composante de certains de ces mythes apolliniens semble se confirmer à propos d'une autre légende. En effet, serait également en rapport avec le motif de la purification dans la vallée de Tempè la version pindarique d'après laquelle Gè voulait précipiter Apollon dans le Tartare à cause de sa violente occupation de Python ${ }^{25}$. Rutherford considère que la prétention de la Terre aurait provoqué un terrible conflit, car le Tartare est le lieu où sont envoyés les ennemis des Olympiens. En réalité, comme on l'a vu à propos de l'Hymne bomérique, le dieu Apollon est présenté parfois comme une menace contre l'ordre Olympien, un thème dont nous aurions ici une simple variation ou adaptation delphique. Quoi qu'il en soit, comme nous l'avons vu à propos du Péan X et de la légende du fleuve Pénéios, il y a chez Pindare des indices de la connaissance de cette tradition, laquelle ne réapparaît ensuite que chez Théopompe, au $\mathrm{IV}^{\mathrm{e}}$ siècle (mais il ne parle que des rapports entre Delphes et la vallée du Tempè) et Anaxandride de Delphes (230-180 av. J.-C.), qui affirme que la $\theta \eta t \epsilon l a$ d'Apollon est due à la mort du serpent delphique. On constate ici une réorientation du motif ancien du châtiment d'Apollon, qui n'était pas en principe lié à la mort de Python ${ }^{26}$.

Si les mythes qui placent la purification du dieu dans les régions du Nord consacrent cette lepà ósós comme parcours apollinien, une autre voie nous mène vers les régions du Sud. Ce parcours alternatif permet d'établir à son tour un fondement des rapports avec la Crète et de renforcer le rôle de la Crète comme dépositaire des plus vieilles traditions religieuses et de pratiques fort anciennes, liées à la religion apollinienne : chant du péan (et les éléments métriques particuliers, les « crétiques » et « péoniens »), techniques de purification, etc. La voie qui conduit en Crète passe d'abord par Sicyone, où est attesté un mythe (probablement lié à un rite initiatique) qui sert d'aition au culte local de Peitho. Après la mort de Python, Apollon et Artémis vont se purifier à Égialée, mais, quand ils passent par un lieu connu comme Pbobos, ils sont surpris par Deîma et ils partent pour la Crète, ce qui va provoquer

23 Nous disposons maintenant de deux ouvrages d'ensemble sur l'Amphictionie delphique : Lefèvre (1998) et SÁNCHez (2001), auxquels je renvoie pour les questions de détail relatives aux premières phases historiques.

24 Rutherford (2001), p. 396-397.

25 Fr. $55 \mathrm{M}$. = H2 R.

26 Cf. Sú́rez de la Torre (1998b). C'est pour cela que je ne partage pas le point de vue de LAMprinoudakis (1999), lorsqu'il applique au voyage apollinien un schéma interprétatif mort/résurrection. Il est très influencé par l'école de Cambridge sur le concept de l'eniautos daimon, mais je trouve son application à Delphes inappropriée. 
une épidémie, une consultation des devins et un rite propitiatoire dont les protagonistes seront sept garçons et sept jeunes filles qui chaque année se rendent au fleuve Sythas pour persuader les dieux par leurs supplications. Dans le lieu de l'apparition de Deîma, on érige un temple. Une fois le dieu arrivé en Crète, nous trouvons des ramifications nouvelles du mythe ${ }^{27}$. Il est purifié par Carmanor ${ }^{28}$ d'après Pausanias, mais une autre version, transmise par l'bypothèse des Pytbiques de Pindare, attribue la purification au fils de Carmanor, Chrysothémis et, pour harmoniser les traditions, ajoute qu'après cette purification, Apollon part pour la vallée du Tempè dont il serait revenu avec le laurier employé pour les couronnes pythiques.

Le travail d'harmonisation est accompagné également de versions évhéméristes. D'après celle que nous transmet Pausanias ${ }^{29}$, le serpent n'aurait jamais existé; par contre, l'histoire véridique serait qu'un Eubéen ambitieux et insolent, Crios, aurait ravagé le sanctuaire et la région, et aurait essayé de renouveler son attaque. Phémonoé aurait émis une prophétie qui annonçait la mort de Crios par les flèches d'Apollon et, ensuite, la purification du dieu par les Crétois.

\subsubsection{Occupation sans combat. Nouvelles versions du mythe de succession}

L'influence d'Athènes à Delphes est évidente, au moins à partir du vi ${ }^{\mathrm{e}}$ siècle av. J.-C. ${ }^{30}$ Le contrôle de cet important centre religieux (avec son poids moral très grand sur les décisions politiques) avait sans doute été un but poursuivi par les plus puissants 'États grecs, et Athènes n'a pas échappé à la règle. Pendant le $\mathrm{v}^{\mathrm{e}}$ siècle, on observe un effort redoublé d'assimilation et de rapprochement des traditions mythiques d'Athènes et de Delphes ${ }^{31}$. L'un des résultats de ce processus est, à mon avis, la création d'une nouvelle version de l'occupation delphique du dieu qui introduit la nouveauté de l'absence de violence, car cette version est commandée par une volonté de souligner l'ordre et la rationalité que représente Apollon ${ }^{32}$. Le premier témoignage se trouve chez Eschyle, dans le prologue de la Prophétesse des Euménides (1 sq.), mais les allusions abondent ensuite. Eschyle établit la séquence Gè Thémis - Phoibé - Apollon, mais les variations abondent, pour aboutir à l'ensemble Gè+Poseidon - Thémis - Apollon qui se trouve chez Pausanias. Eschyle souligne que la succession est pacifique. En opposition avec la ver-

\footnotetext{
27 Cf. Paus., II, 30, 3.

28 Père d'Euboulos, qui est le père de Carma. Celle-ci s'unit à Zeus et donne naissance à Britomartis, dont nous parle Pausanias à propos du temple d'Aphaia à Égine.

$29 \mathrm{X}, 6,7$

30 Cf. Suárez de la Torre (1998d), p. 77-82.

31 C'est ce que reflète l'Ion d'Euripide.

32 Cf. Sourvinou-Inwood (1987), Quantin (1992), SuÁrez de la Torre (1998c). Cf. maintenant l'interprétation à la lumière des théories sur le «Geschlechtergegensatz ", d'un côté, et les rites de passage (VAN Gennep) d'un autre dans Rosenberger (2001), p. 2022 .
} 
sion de l'Hymne bomérique, Apollon arrive sur les côtes de l'Attique et ce sont les Athéniens qui l'accompagnent jusqu'au sanctuaire pour fonder l'oracle. Là-bas, il est accueilli par les Delphiens et leur roi éponyme Delphos ${ }^{33}$.

Comme $M$. Berti le fait remarquer dans ce volume ${ }^{34}$, la présence de Thémis dans le monde de la mantique apollinienne obéit à un effort pour renforcer la légitimité et l'autorité des réponses apolliniennes, en opposition à la tradition qui souligne leur obscurité. Elle est due à des besoins d'ordre politique: Thémis est la mère des Hôrai, des divinités qui ont une double fonction, en tant que déesses des cycles saisonniers en même temps que de l'équilibre civique ${ }^{35}$. De cette façon, on explique aussi la présence de la déesse dans le sanctuaire à partir du v $v^{\mathrm{e}}$ siècle, présence qui renforce les liens avec les traditions athéniennes : elle tient le rôle de la Pythie dans le tondo du Peintre de Berlin (440-430 av. J.-C.) ${ }^{36}$, où elle délivre l'oracle à Égée en $^{37}$.

Comme dans le cas précédent, nous trouvons à nouveau une version de compromis à l'époque hellénistique, dans un effort pour renforcer la logique du récit. Je fais allusion au péan d'Aristonoos ${ }^{38}$, selon lequel, le dieu, après la mort du serpent et sa purification à Tempè, revient avec Athéna, qui persuade Gè et Thémis de céder les droits d'occupation au jeune dieu. Enfin, le thème de la pureté et des procédés pour y parvenir va connaître une dérivation qui atteste une contamination avec les courants orphiques et pythagoriciens. Ce discours religieux est présent dans le dialogue de Plutarque $D e$ defectu oraculorum, quand Cléombrote raconte sa visite au «barbare inspiré » (qui prophétisait une fois par an), lequel parlait des $\pi a ́ \theta \eta$ delphiques de Dionysos et d'Apollon. Il affirmait que l'histoire de Tempè était fausse,

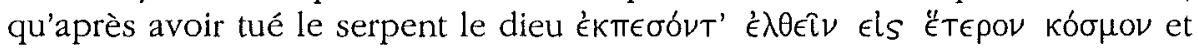
que, après neuf périodes de "grandes années » il revint, devenu vraiment áyvós et $\Phi$ ô̂ßos, pour occuper l'oracle, gardé jusqu'à ce moment par Thémis.

\subsubsection{Apollon et les Hyperboréens}

Partiellement liée à la question de l'occupation du sanctuaire, une autre question concerne les rapports entre Delphes et le pays des Hyperboréens.

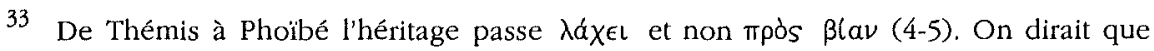
Phoibé est une invention du poète, pour faciliter le passage à Phoibos. Chez Eschyle, Poseidon est cité comme dieu puissant de la région (Eum., 27) mais il est apparemment exclu de la succession.

34 M. BERTI, ici-même, p. 225-234.

35 Cf. les données de BERTI, ibid, à propos des témoignages épigraphiques non seulement de Delphes, mais aussi d'Olympie et de Dodone.

36 Berlin F2538, Antikensammlung, Staatliche Museen, Preussischer Kulturbesitz.

37 Une révision des liens Gè-Thémis et de son évolution à Delphes et Athènes a été faite par STAFFORD (2000), p. 56, qui donne d'autres détails sur les témoignages archéologiques. Elle démontre que Thémis ne peut pas avoir commencé sa « vie » religieuse comme épithète de la Terre, mais que c'est Gaia qui a connu une extension vers le domaine politique (p. 67-68). Cf. aussi Detienne (1998), p. 135-174.

38 Cf. RUTHERFORD (2001), p. 28-29 avec les références. 
Les légendes déliennes sont notre source principale sur la présence hyperboréenne dans un lieu de culte apollinien, mais il ne faut pas négliger les données sur Delphes et, en même temps, ne pas perdre de vue que le lien établi entre ce pays mythique et Apollon a des racines probablement indépendantes et plus générales que les rapports avec un sanctuaire concret. Les traditions relatives à ce peuple légendaire sont abondantes et ont été bien étudiées $^{39}$. En ce qui concerne Delphes, je me limiterai à une indication rapide sur deux points. D'abord, l'originalité (par rapport aux traditions plus connues dans la suite) du témoignage d'Alcée (fr. 307a-b-c V.), dont l'hymne (peut-être un péan ${ }^{40}$ ) qui contenait ce mythe (ou, au moins, la paraphrase en prose conservée d'Himérius) place l'arrivée du dieu au sanctuaire en plein été, tandis que le rituel d'invocation du dieu qui commémorait le commencement de sa présence annuelle avait lieu au printemps, le 7 du mois Bysios. La force de cette tradition locale a poussé certains savants à essayer d'harmoniser diversement le texte d'Alcée avec les autres données, mais on doit être attentif aux remarques de Meyerhoff ${ }^{41}$, lorsqu'il souligne que le chant d'Alcée ne parle pas de la répétition d'une théophanie, mais d'une première arrivée du dieu, car l'épisode se passe immédiatement après la naissance d'Apollon et après l'ordre de Zeus de se diriger vers Delphes. Il s'agit donc incontestablement d'une tradition divergente de celle de l'Hymne bomérique (ce qui n'est pas incompatible avec une fonction étiologique locale). En principe, le

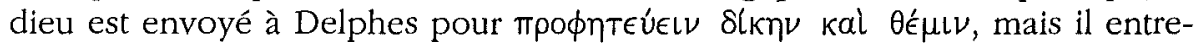
prend d'abord un voyage chez les Hyperboréens, où il passera une année en s'adonnant à l'activité de $\theta \epsilon \mu \iota \sigma \tau \epsilon u ́ \epsilon \iota \nu$. Puis, invoqué par les chants des Delphiens, il arrive au sanctuaire et sa présence est ressentie par la nature entière : la végétation, les eaux (la Castalie et le Céphise sont mentionnés) ainsi que les animaux la détectent. Malgré la difficulté essentielle de fixer précisément-la contribution de l'auteur du résumé par rapport au texte poétique, le poème est un ensemble très révélateur en tant que synthèse de l'idéologie locale, cristallisée cette fois sous forme lyrique.

Deuxièmement, dans le récit d'Himérius, un fait singulier attire notre attention: Apollon n'obéit pas à Zeus. On dirait qu'ici reparaît un vieux thème du monde religieux grec : le conflit entre générations divines. L'hymne d'Alcée symbolisait une sorte de rébellion pacifique du nouveau dieu qui veut établir un domaine propre indiscutable. En réalité il s'agit de la rationalité du mythe qui doit expliquer l'existence d'un pays apollinien (ou transformé comme tel par la tradition) qui accueille le dieu périodiquement et pendant des périodes de crise olympienne. En effet, au chant IV des Argonautiques d'Apollonios de Rhodes, on mentionne une légende celte d'après laquelle les eaux de l'Éridan sont en réalité les larmes versées par Apollon

\footnotetext{
39 Cf. les références dans Suárez de la TORre (1998b), p. 316-319.

40 Cf. Rutherford (2001), p. 27-28. Trois sources le décrivent comme un péan, mais d'autres comme bymne et prooimion.

41 MeYerhoff (1984), p. 162 sq.
} 
pleurant la mort d'Asclépios, tué par Zeus pour avoir ressuscité des mortels,

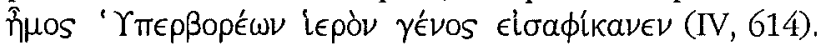

En tout cas, cette version d'Alcée n'annule pas (mais renforce et enrichit) l'insistance mise sur le concept de mouvement et de déplacement fondateur qui ressort des autres versions d'occupation. Toujours le voyage, toujours le pèlerinage. Cependant, comme s'il s'agissait d'une sorte de « théologie du voyage apollinien », les étapes intermédiaires entre Délos et Delphes sont modifiées et dans leurs fonction et dans leur nature. La première partie soulignait l'acquisition des attributs externes du dieu. Puis l'hymne marquait une étape dans le domaine eschatologique du dieu, qui alterne indéfiniment avec le séjour delphique : l'activité de la thémis oraculaire du sanctuaire trouve une correspondance dans le pays merveilleux où séjourne le dieu.

\subsection{L'équilibre dans le panthéon local ${ }^{42}$}

Un groupe important de mythes est destiné à rendre vraisemblable l'existence d'un culte de certains dieux différents d'Apollon ou bien à légitimer le pouvoir apollinien sur certaines sphères qui pourraient être de la responsabilité des autres dieux.

\subsubsection{Apollon et Dionysos. Les rites et les mythes dionysiaques}

Le mythe de l'alternance de la présence dans le sanctuaire des deux fils de Zeus signale le trait le plus représentatif du panthéon delphique. Or, il ne s'agit pas d'un équilibre parfait. Le dieu oraculaire l'emporte sur le dieu de la transe, dont la fonction prophétique n'est mentionnée que très rarement: une des bypothèses des Pythiques de Pindare dit que Dionysos avait été le premier à prophétiser à Delphes, un thème qui pourrait avoir servi parfois d'argument au nomos pythique, car ce mythe est cité par l'auteur de l'bypothèse à propos d'une partie de ce type de composition ${ }^{43}$. En tout cas, le dieu de Delphes est sans discussion Apollon. Ce phénomène a provoqué probablement une présence plutôt limitée de mythes dionysiaques delphiques, mais les rituels et quelques témoignages de nature diverse sont indiscutables. Laissant de côté la question des origines du Dionysos delphique ${ }^{44}$, les rites et les mythes confirment la parfaite intégration du dieu dans l'enceinte apollinienne : que ce soit les renseignements sur les rites dans les sources littéraires (cf. le rituel de l'Héroiss et le mythe de Sémélé/Thyôné, les rites orgiastiques dans le Parnasse ou dans l'antre Corycien, ou bien la tradition que nous connaissons avec des traits ouvertement orphiques, repris par la tradition tardive chrétienne, de la mort $d u d i e u^{45}$, enseveli dans le temple

42 Pour un traitement détaillé de la question je renvoie à Suárez DE LA TORRE (1998d).

43

Pyth. Hypoth. (a) vol. II, p. 2 Dr. Cf. SuÁrez de la Torre (1998a).

44 Cf. Dietrich (1992).

45 En principe, démembré par les Titans et enseveli par Apollon, après avoir réuni ses membres. Le rapport avec la tradition argienne de la mort du dieu aux mains de Persée est une adaptation chrétienne très tardive : $c f$. les précisions de PiÉrart (1996). 
apollinien et éveillé par les Tbyiades) ou les témoignages épigraphiques et iconographiques, ou même le fait qu'un péan soit composé au Iv siècle en l'honneur de Dionysos ${ }^{46}$. Tout cela manifeste un effort d'harmonisation entre ces dieux qui a des parallèles par toute la Grèce.

\subsubsection{Les autres dieux du sanctuaire}

Pour les autres dieux liés au sanctuaire, on observe un décalage remarquable entre certains mythes et la présence des ces dieux dans la réalité matérielle du sanctuaire. Si Athéna ou Artémis, par exemple, disposent physiquement d'une partie de l'ensemble architectural, ce n'est pas le cas des autres dieux ${ }^{47}$. Poseidon ou Hestia, de leur côté, doivent se contenter d'un autel, et les archéologues ont des doutes quant à la vraie identité des vestiges de la « chapelle de Gè » ou de la «fontaine des Muses », bien que l'épigraphie ne laisse pas de doutes quant à l'existence de ces cultes dès le vi ${ }^{\mathrm{e}}$ siècle av. J.-C. Les mythes sur Léto sont bien représentés partout en Grèce, mais le seul monument local est le « rocher de Léto » (d'ailleurs douteux). Autrement dit, à Delphes, abondent les mythes sans correspondance matérielle ${ }^{48}$. Ils se sont développés librement, pour illustrer des concepts ou pour établir un cadre cohérent des rapports entre les dieux. Ils composent un modèle flexible, qui varie suivant les intérêts de chaque époque, sans exclure la coexistence de plus d'une version: Eschyle s'efforce de prôner un modèle de succession pacifique, très efficace dans le cadre idéologique des Euménides, mais les concours musicaux des Jeux Pythiques évoquent obstinément la pythocbtonie, le grand mythème local.

Il est des dieux sans temple (apparemment), mais dont la présence se laisse pressentir souvent. Ainsi, par exemple, Hermès. L'Hymne bomérique IV exemplifie la résolution d'un conflit entre deux dieux de la médiation, de la communication, et trace des frontières nettes pour expliquer une harmonie divine qui aurait pu être sérieusement menacée. En tout cas c'est un équilibre qui embrasse plusieurs aspects de la musique et de la mantique, car Hermès n'est pas dépourvu d'un certain rôle dans ces deux domaines, et qui se traduit parfois en une opposition « complémentaire ». Le paradigme de cette opposition est fixé dans la légende du poète mythique Philammon. Il est fils d'Apollon et de la nymphe Philonis, la fille de Déion, laquelle concevra d'Hermès un autre fils, Autolycos, qui héritera, comme l'a signalé Pòrtulas ${ }^{49}$, les traits de trikcster de son père, car il deviendra un voleur modèle. Pour sa part, Philammon sera un extraordinaire musicien, vainqueur des premiers concours musicaux, mais aussi une sorte de héros local, car il mourra pendant un combat contre les Phlégyens, qui attaquaient Delphes.

46 Pour les détails, je renvoie à nouveau à SuÁrEz DE la TORRE (1998a).

47 Il y a aussi des dieux avec un temple, mais sans mythes delpbiques. Ce sera, par exemple, le cas d'Asclépios.

48 Cf. le cadre tracé par AMANDRY (2000).

49 Pòrtulas (2000). 


\section{Les héros}

Cette catégorie, typiquement grecque, est fondamentale et indispensable pour articuler un discours religieux complet. Évoluant entre l'humanité et les dieux $^{50}$, héros et hérö̈nes deviennent des éléments substantiels du code qui vise à rendre compte des noms, des traditions et même des valeurs et principes qui soutiennent l'ensemble des croyances. Les groupes que j'ai établis répondent à la fonction des héros dans l'entourage delphique et aux traits définis par les récits, de la simple éponymie à une fonction plus complexe (comme illustration de principes moraux) ou à la protection du sanctuaire. Il en résulte un cadre fonctionnel semblable à celui des autres cités grecques, mais avec certains traits particuliers, surtout en ce qui concerne la fonction paradigmatique des principes et des traditions delphiques. Je ne vais pas traiter les mythes qui ont comme motif décisif une consultation oraculaire affectant directement un héros (surtout les héros fondateurs), car ce thème exige une recherche particulière d'une nature différente ${ }^{51}$. Je me limiterai aux héros dont certaines actions sont liées au sanctuaire delphique.

\subsection{Héros éponymes et "généalogiques » de Delpbes}

D'après L. Lacroix la liste des éponymes, trasmise surtout par Pausanias, « est le produit d'une érudition qui aimait à reconstituer un passé légendaire, à l'aide des traditions locales $»^{52}$. Sa rigoureuse analyse du texte de Pausanias, complétée par la référence à d'autres sources, me permet de ne pas m'arrêter à ces questions. Le maître liégeois a établi clairement les deux principes qui président à la description du Périégète. À la tendance géographique s'ajoute une autre, étymologique (ici appliquée vraiment "en cascade »). Le cadre présenté dans l'Appendice résume clairement ces généalogies et permet d'apprécier ces traits. On trouvera également dans ce cadre un schéma qui réproduit la généalogie de la scholie au vers 1094 de l'Oreste d'Euripide, analysée récemment par Sánchez ${ }^{53}$ à propos des origines de l'Amphictionie. Il observe à cet égard une fusion entre deux traditions contradictoires. En réalité il s'agit d'un tissu assez complexe qui (seulement du point de vue généalogique ${ }^{54}$ montre un mélange des versions de Pausanias qui reçoivent les numéros 2, 4 et 6 dans l'Appendice.

\subsection{Les béros "batisseurs ": Trophonios et Agamédès}

Pausanias mentionne un poème de la poétesse Boiô (inconnue ailleurs) où, dans l'ensemble d'une relation de plusieurs personnages Hyperboréens,

50 Pour une révision récente des problèmes concernant la figure du héros $c f$. Pirenne-Delforge \& SuÁrez de la Torre (2000).

$51 C f$, par exemple, la méthodologie suivie par Rurz PÉrEz (1995).

52 Lacroix (1994), p. 274.

53 SÁNCHEZ (2001), p. 60-63.

54 Je n'étudie pas ici les questions abordées par SÁNCHEZ, auquel je renvoie pour les détails de l'effort de justification de l'antiquité mythique de l'Amphictionie. 
on attribuait à deux héros de cette origine, Pagasos et Agyieus (un sios 'A $\gamma u$ utés sous lequel semble se cacher le dieu en personne) la fondation du $\chi \rho \eta \sigma \tau \operatorname{r}^{\prime} \rho \nu^{55}$. On ne pourrait préciser l'antiquité de cette tradition, mais en tout cas elle doit être mise en rapport avec le mythe de la succession des temples du sanctuaire. Le Péan VIII de Pindare (B2 Rutherford) est le texte le plus ancien qui le mentionne ${ }^{56}$. Ce texte parle de quatre temples bien différenciés. Pausanias ajoute une référence au temple construit par des mains humaines, après celui des héros fils d'Erginos (Trophonios et Agamédès), ce qui prolonge l'histoire jusqu'à un niveau historique plus proche ${ }^{57}$. L'importance de ce récit est indiscutable, d'un côté, en tant que réflexion issue d'une perspective évolutionniste morcelée sur le binôme nature/culture (comme l'a démontré Sourvinou-Inwood ${ }^{58}$ ) et, d'un autre, en tant que paradigme symbolique des différents niveaux de l'activité essentielle du sanctuaire, c'est-àdire de la mantique ${ }^{59}$.

Mais le mythe des temples et, surtout, le rôle de Trophonios et Agamédès, nous renseignent sur d'autres aspects. $P$. Bonnechere ${ }^{60}$ a fait une analyse très fine des légendes sur Trophonios et Agamédès qui nous permet d'approfondir la connaissance de la figure du héros en général et des héros delphiques en particulier. Chez Pindare ${ }^{61}$, on soulignait l'aspect de la mort des héros comme rémunération pour avoir construit le temple. Exprimé de cette

55 Paus., X, 5, 8 : Пєтоl̆

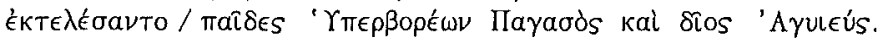

56 Cf. RuTherFord (2001), p. $210 \mathrm{sq}$, avec un commentaire très important.

57 Paus., X, 5, 9-12. Mais il suprime l'un des temples : il aurait dû parler de six. $C f$. aussi des références (moins détaillées) à la légende dans Aristote, fr. 3 Rose; Strabon, IX, 3, 9; Philostrate, Vita Apollonii, 6-11.

58 Sourvinou-INwOOD $(1979=1991)$.

59 Il va de soi que Pindare « may have been exploiting traditional ideas about divine architecture reflected in rituals, architecture, or other myths $»$ (RUTHERFoRd 2001, p. 225), mais c'est seulement un moyen de transmettre un message assez plus complexe. L'analyse anthropologique de ce récit peut être approfondie en tant que réflexion sur la mantique delphique, surtout au départ de la version pindarique qui présente des éléments

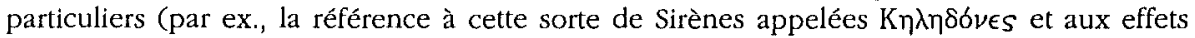
de leurs chants). Vu que, après le niveau primordial, il s'ensuit une évolution dans laquelle s'introduit un élément apparemment négatif (au moins «trompeur »), on dirait que ce qui est illustré dans cette version est le besoin d'arriver à un stade dans lequel la transmission du message émis par les dieux n'implique pour les hommes ni le danger du mensonge ni l'envoûtement séducteur. On pose donc le besoin et d'une médiation qui a des conséquences positives pour l'humanité et de la construction d'un bâtiment qui symbolise le nouvel état. Je pense qu'il y a dans la présentation pindarique une démonstration de la providence apollinienne et de sa sagesse infaillible. Le nouveau temple va accueillir la prophétie de la parole vraie, proclamée à travers les oracles donnés à Erginos. Les constructeurs de ce nouveau temple auront une «biographie » pleinement conditionnée par le dieu Apollon, depuis leur naissance, jusqu'à la mort.

60 BONNECHERE (1999).

61 Pind, fr. 2-3 Maehler. 
façon, on pourrait penser à une amère ironie du dieu. Bonnechere a raison : il faut chercher le côté positif. Et celui-ci n'est autre que la perspective d'immortalité et d'activité (oraculaire et médicale) dès l'au-delà réservé à Trophonios. En revanche, l'auteur propose que le témoignage de l'Hymne bomérique à Apollon (la suite Pytbique, v. 294-299), où il est question de la construction du lainos oudós par les deux frères après qu'Apollon en a jeté les fondations, et la comparaison avec les autres oeuvres architecturales de ces héros font penser à une forte charge sémantique du substantif seuil, pleine de connotations dans le cas de ces héros qui se placent dans un espace de communication réglée avec les dieux (des héros de «la limite »). Je voudrais ajouter à cette séduisante interprétation l'importance, une fois encore, des rapports entre l'activité des héros et celle du dieu delphique: divination et construction. Trophonios continue le travail de fondation entrepris par le dieu et, après la mort, il devient un héros aux pouvoirs mantiques. Une nouvelle démonstration de la consistance du 'système' héroïque créé autour de Delphes.

\subsection{Conflit et conciliation (ou "la violence et le sacré »)}

Autour de trois héros se sont développés des mythes qui constituent une vraie réflexion sur une question fondamentale de la religion delphique: l'expiation d'un crime de sang.

\subsubsection{Héraclès ${ }^{62}$}

Une série importante de récits mythiques nous présente Héraclès en tant qu'allié du dieu delphique. Nous avons déjà mentionné son rôle dans le processus de "nettoyage » de brigands de la région et sa collaboration comme allié d'Apollon. Or, le héros thébain est également le sujet d'une histoire qui exemplifie un conflit où se confondent le problème de la purification d'un crime et celui de l'autorité mantique. Ayant commis le meurtre d'Iphitos, fils d'Eurytos (soit à cause d'un rapt de folie, soit consciemment) ${ }^{63}$, Nélée, épouvanté par la brutalité et la démesure du crime ${ }^{64}$, refuse de purifier Héraclès, ce que fera finalement Déiphobe. Malgré la purification, Héraclès tombe malade et se rend à Delphes pour obtenir un oracle guérisseur. La Pythie s'y oppose, ce qui déclenche la colère d'Héracles qui ravage le temple, prend le trépied et prétend prophétiser lui-même. Apollon lui dispute la possession du trépied et Zeus doit mettre fin à la querelle en employant la foudre. Puis, il oblige la Pythie à prophétiser : l'oracle aura comme consé-

62 Un bon résumé des mythes delphiques d'Héraclès se trouve dans Defradas $\left(1972^{2}\right.$ ), p. 123-146 (liste des sources p. 157-159).

63 Hom., Od. XXI, 22-30; Apollod., II, 6, 1-2; schol. BQ à Homère; Phérécxde (3 F 82b Jácoby); SopHocle (Tr., 262-279) et D.S. 431, 2-3.

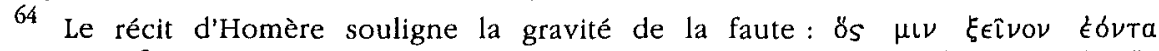

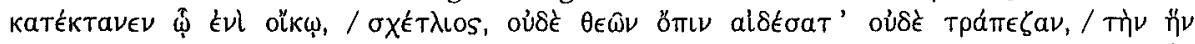

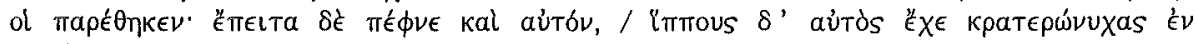

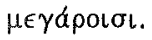


quence la période de servitude d'Héraclès comme esclave de la reine Omphale de Lydie. Zeus rétablit donc l'équilibre et l'autorité mantique du sanctuaire, mis en cause par le plus violent des héros.

\subsubsection{Oreste et Néoptolème}

Envoyé furtivement par Électre en Phocide (après la mort d'Agamemnon) quand il était encore un enfant, Oreste, une fois adulte, consultera l'oracle apollinien sur la vengeance contre les assassins. Apollon ne laisse aucun doute sur la justification de cette vengeance, mais insiste davantage sur la nécessité de la purification, qu'il commence aussitôt à Delphes. Ce mythe a connu des analyses exhaustives, surtout dans le cadre de l'Orestie d'Eschyle et à propos des tragédies d'Euripide, analyses auxquelles je renvoie pour un traitement plus approfondi ${ }^{65}$. En tout cas, retenons l'importance du problème moral posé, car c'est le dieu de la purification qui permet le crime à l'intérieur du génos. Eschyle avait, donc, une bonne base pour innover et en profiter ad maiorem Athenarum gloriam, ce qui ne signifie point que, dans la tradition épique, les actes d'Oreste n'étaient pas appréciés dans cette dimension inquiétante. Parker a raison de souligner que, si Homère réussit à donner une image positive est noble d'Oreste, c'est au pris d'une insistance sur le crime d'Égiste et d'un silence sur la mort de Clytemnestre ${ }^{66}$.

Oreste est lié par d'autres motifs au héros qui a son tombeau dans le sanctuaire, Néoptolème. Sous les récits (parfois certainement romanesques) crées depuis le cycle épique et les diverses versions du mythe qui expliquent la mort de Néoptolème (dont les auteurs sont Oreste ou les prêtres delphiques ou le dieu en personne), Fontenrose pensait que se cachait le mythe bien différent du combat entre anciennes divinités qui représentaient, respectivement, l'hiver et l'été, la mort et la vie, etc. ${ }^{67}$ Or, toute proposition " évolutionniste " doit se poser d'abord la question de savoir à quel moment de l'histoire delphique a pu avoir lieu un tel processus. Faute d'une réponse précise, il vaudra mieux de se contenter d'une interprétation des mythes plus strictement liée à l'activité et aux cultes du sanctuaire. Dans cette ligne, Usener $^{68}$ (qui, néanmoins, était aussi prêt à y voir des rites anciens liés à la succession des saisons) avait avancé déjà quelques propositions intéressantes et, depuis lors, cette dernière interprétation a été la tendance majoritaire de la recherche, que ce soit à partir de la dichotomie theós/beros et dans le cadre d'un procès d'intégration du rôle du héros dans un système polythéiste (Chirassi Colombo ${ }^{69}$ ), ou par contraste avec le culte normal des héros, à cause du panhellénisme du personnage (un développement auquel la tradition épique n'est point étrangère) et avec un rapport très direct avec les 
pratiques cultuelles de Delphes $\left(\mathrm{Nagy}^{70}\right)$. Pour ma part, j'ai essayé ailleurs de contribuer à l'interprétation de ce culte delphique en tenant compte autant des données épigraphiques que des textes littéraires et en plaçant le rite dans le cadre des fêtes locales et de leurs modalités ${ }^{71}$.

\subsection{Ion et la mytbologie atbénienne}

Chez Eschyle la modification du mythe vise à reserrer les liens entre Delphes et Athènes par l'introduction d'un nouveau parcours d'arrivée du dieu. Euripide va encore plus loin. Cette fois il s'agit de modifier une généalogie pour créer un lien entre le dieu et les Érechthéides : Ion n'est plus le fils de Xouthos et Créuse, mais de celle-ci et d'Apollon. D'un côté, N. Loraux ${ }^{72}$ nous a laissé un modèle d'analyse de cette œuvre qui montre bien à quel point elle est une "mise en scène de l'autochtonie » dans un entourage delphique. On est arrivé à considérer Delphes comme un simple prétexte pour déployer cette reconstruction de la mythologie athénienne ${ }^{73}$. Mais, d'un autre côté, on peut défendre la perspective complémentaire : il s'agit plutôt d'une interprétation des réalités delphiques à l'aide d'une clef athénienne.

\subsection{Les béros bomériques à Delphes: Agamemnon, Acbille, Ulysse.}

Delphes fait déjà partie du «paysage homérique ». Achille n'accepterait jamais la compensation qu'Agamemnon lui propose même si elle était aussi riche et abondante que les trésors du sanctuaire apollinien ${ }^{74}$. Démodoque évoque un $v \in \hat{\imath} \kappa o s$ entre Achille et Ulysse ${ }^{75}$ qu'Agamemnon interprète comme accomplissement d'un oracle qu'il avait reçu à Delphes ${ }^{76}$. Enfin, le Parnasse est la région dans laquelle a lieu la chasse du sanglier qui va provoquer la blessure d'Ulysse dont la cicatrice permettra sa reconnaissance par Euryclée $^{77}$. Richesse des offrandes, prophétie mêlée à l'action épique, le Parnasse

70 NAGY (1979), p. 118-141.

71 Sú́rez de la ToRre (1997); $c f$. aussi WoOdBury (1979). Il faut ajouter maintenant à la bibliographie Rutherford (2001), p. $315 s q$.

72 "Créuse autochtone", dans Loraux $\left(1990^{2}\right)$, p. 197-253.

73

OWEN (1939), p. XXII.

74 Il. IX, $404 s q$.

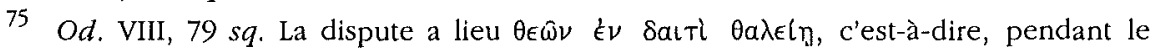
banquet qui suit la célébration d'un sacrifice.

76 Fernández Delgado (1991), suivant une interprétation des scholies, considère qu'Homère adapte la tradition de l'ambigüité des oracles : Agamemnon l'interprète faussement. Pour NAGY (1979), 118-141, les liens entre Achille, Delphes et la dais dans cet épisode sont appropriés en tant que « an opening Strife Scene for motivating the eventual death of the main hero who undertook the enterprise " (p. 141). En fait il s'agit d'une allusion à un épisode de la tradition épique perdue dans lequel le motif de l'ambiguité oraculaire n'aurait aucun rôle. Or, le problème principal est de localiser cet épisode dans l'action épique, car il doit être antérieur à l'arrivée des Achéens à Troie (plus exactement : antérieur au départ de la Grèce), car nous ne connaissons aucune " absence » d'Agamemnon pendant la guerre (mais elle n'est pas exclue non plus).

77

Od. XIX, $392 s q$. 
comme paysage initiatique du jeune héros... Somme toute, un petit " rameau d'or » delphique.

\subsection{Les protecteurs du sanctuaire}

Pour un lecteur espagnol, l'aide prêtée par l'équivalent chrétien du héros, c'est-à-dire un saint, au moment d'une invasion des ennemis de la foi catholique, n'est pas un thème étrange, car l'image de Saint Jacques « Matamoros » brandissant son épée contre les musulmans lui est tout à fait familière. Le mythème est attesté partout et l'histoire de Delphes n'y échappe pas. Premier épisode, raconté par Hérodote ${ }^{78}$, l'invasion perse : surprise désagréable pour l'ennemi, qui est victime des manifestations de la colère divine. La foudre tombe sur eux, les rochers des montagnes les écrasent et, du sanctuaire d'Athéna Pronaïa, arrive un terrible cri de guerre. Pour renforcer la défense,

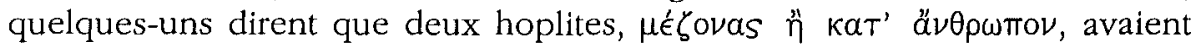
poursuivi et tué un grand nombre de guerriers : c'étaient Pbylaque et Autonoos, dont les $\tau \in \mu \epsilon ́ v \in \alpha$ étaient situés, respectivement, près du temple de la Pronaïa et à côté de la source Castalie. Deuxième épisode : l'invasion des Celtes, commandés par Brennos (279 av. J.-C.). Cette fois c'est Pausanias ${ }^{79}$ qui fait le récit. La panique tombe à nouveau sur l'ennemi, cette fois à cause d'une série non moins impressionnante de prodiges : tremblements de terre, tonnerres, foudre incessante et, une fois encore, le fantôme des héros protecteurs. Mais les temps changent et, cette fois, ceux qui étaient en service de garde s'appelaient Laodochos et Hypérochos (donc, deux Hyperboréens ${ }^{80}$ ), auxquels quelques-uns, dit Pausanias, ajoutent le nom de Phylaque.

\section{Les médiateurs entre les hommes et les dieux}

Il va de soi que les héros sont à juste titre une catégorie de médiateurs. Cependant, j'ai préféré établir une division spécifique, composée de tous ceux qui jouent un rôle déterminé dans le procès strict de la communication verbale entre les hommes et les dieux, un groupe essentiel à Delphes. À noter l'importance de la femme dans cet ensemble.

\subsection{Pythies, prêtres, poètes et poétesses}

Après avoir donné des renseignements sur les villes voisines et les chemins qui conduisent au sanctuaire et avant de commenter son déplacement à l'intérieur de l'enceinte, Pausanias donne un résumé de l'histoire locale en

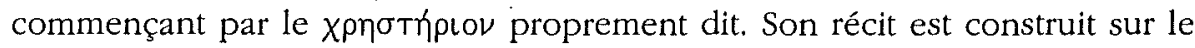
modèle évolué qui avait triomphé depuis le $\mathrm{vi}^{\mathrm{e}}$ siècle. Le schéma employé est évident : succession des divinités propriétaires accompagnées des noms des

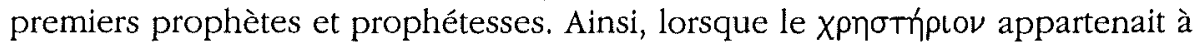
Gè, la première mpó $\mu a \nu$ Tıs avait été Daphnis, l'une des nymphes des monta-

\footnotetext{
78 HÉRoDOTE, VIII, 37-39.

$79 \mathrm{X}, 23,1-3$.

80 Masculin de deux des vierges hyperboréennes de Délos chez Hérodote, IV, 35.
} 
gnes voisines. Des vers tirés de l'Eumolpie de Musée (à remarquer le poids de la tradition fabriquée par les Athéniens) l'obligent à expliquer que, à côté de Gè, Poseidon partageait le sanctuaire et que son prêtre était Pyrcon. Puis il raconte comment Apollon succède pacifiquement à Thémis et explique que Poseidon avait reçu en compensation le sanctuaire de Calaurie, près de Trézène. La mention des émanations d'un àTuós qui avaient provoqué la transe mantique des bergers ${ }^{81}$ sert à introduire le nom de la première

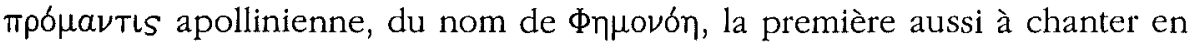
hexamètres. Néanmoins, il connaît un poème de la poétesse Boiô qui contenait la version hyperboréenne (citée supra) des origines du temple, fondé par Pagase et Agyieus. Le poème de Boiô énumérait d'autres Hyperboréens, y compris Olen, qui aurait été le premier mpoфátas d'Apollon. Enfin, Pausanias précise que, à cette exception près, on ne connaît aucun autre homme dans cette fonction mantique. Puis, il introduit le mythe de la succession des temples.

Sous l'inévitable couche érudite, on aperçoit bien le principe qui préside à cette description, qui d'ailleurs s'accorde avec le mythe des temples. Pausanias dispose de textes poétiques qui reflètent des tendances chronologiquement et idéologiquement diverses et il essaie de les harmoniser avec une évidente logique évolutionniste. La première divinité, chthonienne par antonomase, a comme servante une Nymphe, une figure qui synthétise les forces de la nature, le niveau primordial d'inspiration à travers les éléments liés à la terre, surtout l'eau, qui aura toujours un rôle substantiel dans le procès de communication mantique à Delphes, que ce soit pour la purification préalable dans la source Castalie, ou pour le renforcement du pouvoir prophétique grâce à la source Cassotis. La force mantique et inspiratrice qui provient de la terre a coexisté toujours avec la force d'inspiration proprement dite (du point de vue étymologique), c'est-à-dire pneumatique dans les sanctuaires apolliniens. Or, on perçoit une forte tendance à établir des niveaux bistoriques. Gè est accompagnée par Poseidon dans ces fonctions

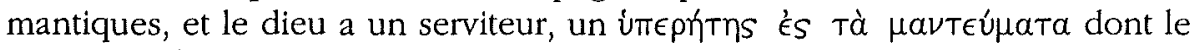

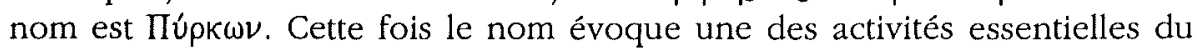
sanctuaire : le sacrifice. Le « sacrificateur » de Néoptolème porte le nom de Maxaıpєús. Les noms aident donc également à construire un réseau sémantique enraciné dans les procédés du manteion.

Puis, Pausanias construit habilement le passage au stade apollinien, caractérisé par un registre fortement verbalisé, mais qui n'exclut pas des éléments des strates précédentes. Les traditions qu'il emploie sont parfaitement liées, malgré leurs origines plutôt disparates : la succession pacifique permet le passage à une période apollinienne liée au mythe des Hyperboréens ( $c f$. cidessus). D'abord, une prépondérance, dans cette phase, du message oral sous forme poétique, représenté par Phémonoé. Comme je l'ai fait remarquer ci-dessus, la fondation du temple par Pagasos et Agyieus peut être harmo- 
nisée avec la légende de la succession des temples, qui est représentative d'un ensemble idéologique complexe autour de la nature du dieu et de son oracle. À la même tendance obéit le rôle d'Olen, un nouveau paradigme de la nouvelle phase, dans laquelle la parole poétique-propbétique est décisive.

Les noms de Musée et d'Olène nous mettent en contact avec un ensemble très représentatif de la culture à la fois religieuse et littéraire des poètes mythiques grecs ${ }^{82}$. Avant de revenir à ces poètes, ajoutons deux autres noms du même groupe qui ont des rapports avec Delphes : Philammon et Thamyris. Sur le premier, je renvoie aux idées déjà exprimées ci-dessus ${ }^{83}$. Une victoire musicale pythique est attribuée aussi à Thamyris (et on pourrait aussi rappeler le nom de Chrysothémis, le fils de Carmanor). Quant aux deux poètes que cite Pausanias, c'est surtout Olen qui nous intéresse. Il s'agit d'un nom strictement lié à la religion apollinienne et, en principe, au sanctuaire de Délos (où il est présenté comme un Lycien). Sa présence dans une tradition delphique (la source est la poétesse locale, par ailleurs inconnue et, probablement, de date tardive, Boiô) a l'air d'obéir à un effort pour équilibrer les légendes de tendance hyperboréenne entre les deux pôles de la géographie apollinienne, Délos et Delphes, mais, plus important encore, elle s'accorde très bien avec le principe fondamental des légendes delphiques sur les poètes et la poésie : rattacher le sanctuaire aux origines de la poésie et de la musique, consacrer l'omphalos en tant que point de départ de la parole inspirée dans tous ses dimensions (poésie, oracle, sagesse, moralité). En somme, le principe et la quintessence de la parole révélée. Le même principe est à la racine des légendes relatives à la présence des Muses à Delphes ${ }^{84}$.

Sous cet éclairage deviennent parfaitement «logiques » les histoires concernant les deux grands poètes de la Grèce ancienne, Homère et Pindare. La vérité historique, comme d'habitude, devient moins importante que le message transmis. À un moment donné (comme Pausanias nous en informe), une image en bronze d'Homère a été placée dans le pronaos du temple apollinien sur une stèle : on pouvait y lire l'oracle reçu par le poète et qui prédisait sa mort ${ }^{85}$. La Vita attribuée à Plutarque ${ }^{86}$ ajoute une version plus longue de l'oracle et explique les circonstances de la fin du grand poète, semblable à la défaite fatale de Calchas contre Mopsos. Dans ce cas, Homère n'est pas vaincu, mais il n'est pas capable de résoudre l'énigme des enfants-

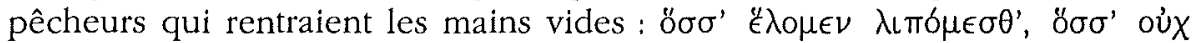

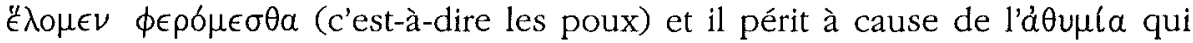
provoqua son ignorance. Le choix de l'oracle qui avertissait Homère illustrait la supériorité de la sagesse et de la prévision delphiques par rapport à

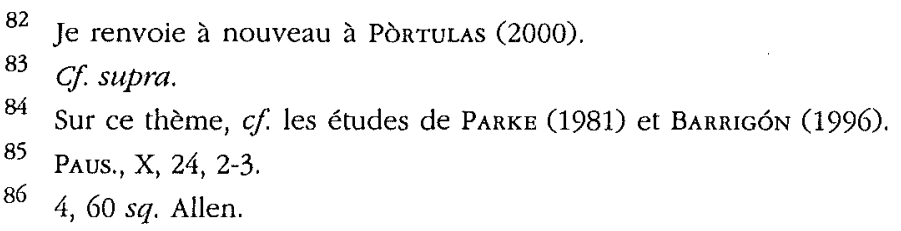


l'inspiration du plus grand poète de la Grèce. C'était une bonne garantie, que les consultants pouvaient lire avant de pénétrer dans le temple pour la session mantique. Puis, à l'intérieur, ils contemplaient, à côté des signes de la présence divine et héroïque, le trône de Pindare et, sans doute, pouvaient-ils être renseignés sur les liens entre le Thébain et le sanctuaire (il avait le droit de recevoir une portion des offrandes, comme un prêtre) et entendre comment le prophétès, avant de fermer le temple, invitait le poète, d'une voix

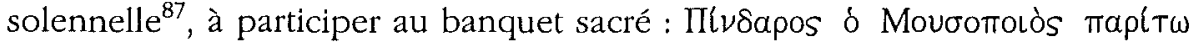

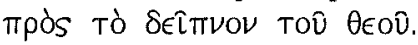

Avant de passer aux Sibylles, je vais ajouter une remarque à propos d'un autre personnage delphique, une femme cette fois. Les informations précises qui la concernent ne sont pas très abondantes et racontent souvent des épisodes négatifs ou tragiques (cas de fraude ou de mort dans des circonstances dramatiques) ${ }^{88}$. Néanmoins il est un exemple très différent qui peut s'expliquer parfaitement par la conjonction de deux circonstances : le rôle des femmes delphiques dans le procès de transmission de la parole révélée et la tendance à faire de Delphes une sorte de foyer de la sagesse. Aristoxène (selon Diogène ${ }^{89}$ et Porphyre ${ }^{90}$ ) affirmait que Pythagore enseignait des choses qu'il avait apprises d'une femme delphique (qu'ils appellent respectivement Themistocléa et Aristocléa) et qu'il expliquait une partie de cette doctrine

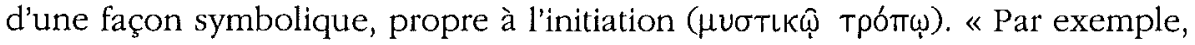
qu'il appelait la mer 'larme', les Ourses 'mains de Rhéa', les planètes 'chiens de Perséphone', et que l'écho produit par la percussion d'un objet en bronze était la voix d'un démon renfermé là-dedans ${ }^{91}$. Indépendamment de l'enseignement de cette femme de Delphes, la biographie de ce philosophe foisonne d'éléments apolliniens remarquables. D'après une version locale samienne ${ }^{92}$, Pythagore aurait été fils d'Apollon et de Pythaïs. Jamblique, qui nous renseigne sur ce point, laisse entrevoir qu'il s'agit d'une version malicieuse transmise par Épiménide, Eudoxe et Xénocrate ${ }^{93}$, mais il admet

87 C'est la version de la Vita Ambrosiana. Il y a une variante dans la Vita de Thomas Magister: Pindare recevait une portion des sacrifices à Apollon; c'était le prêtre qui, $k v$

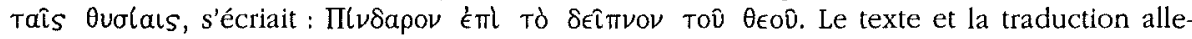
mande de ces Vitae peuvent être consultés dans l'édition d'O. Werner, Pindar. Siegesgesänge und Fragmente, München, E. Heimeran, s.a. (Tusculum-Bücher).

88 Cf. Roux (1976), p. 64-70.

89 Vita Pyth., 8.

90 Vita Pyth., 41 (mais celui-ci ne mentionne pas la source).

91 PORPHYRE, l.c.

92 Iambl., Vita Pyth, 5-8 cite même deux vers d'un toıntrís tLs samien.

93 Le père de Pythagore, Mnémarque, aurait reçu une réponse delphique qui annonçait la naissance d'un fils extraordinairement doué, qui eut lieu en Syrie. Alors Mné-

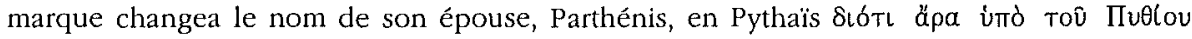

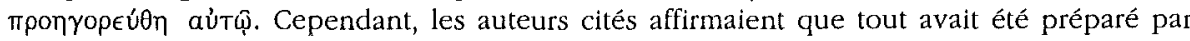
Apollon, car il était le vrai père de l'enfant. 
que l'âme de Pythagore provenait du cortège céleste d'Apollon ${ }^{94}$. Le plus surprenant est l'information transmise par Porphyre ${ }^{95}$, selon laquelle Pythagore aurait composé une épigramme pour le tombeau d'Apollon (fils de Silénos) à Delphes, car le dieu avait été tué par Python et enseveli dans le trépied, nom qu'il dérivait du fait que les trois filles de Triopas auraient chanté le tbrênos d'Apollon ${ }^{66}$ : il va de soi qu'il fallait faire mourir le dieu pour soutenir la légende de l'incarnation apollinienne de Pythagore ${ }^{97}$, sous la forme chère aux Crotoniates, qui l'appelaient Apollon Hyperboréen et aussi Pythique et Péan ${ }^{98}$.

\subsection{Sibylles}

Le rocher de la Sibylle amène Plutarque et Pausanias à déployer leurs connaissances au sujet de la tradition locale concernant cette figure prophétique. Plutarque ${ }^{99}$ cite plus d'une version. La première signale l'Hélicon comme lieu de provenance de la Sibylle, où elle aurait été nourrie par les Muses (le Sacro Parnaso de Calderón avait de glorieux précédents!). La seconde dit qu'elle venait du pays des Maliens et qu'elle était fille de Lamie (la petite-fille de Poseidon) ${ }^{100}$. Cette version est connue de Clément d'Alexandrie, qui ajoute le témoignage d'Héraclite (ce qui a fait penser que la Sibylle citée par le philosophe était celle de Delphes, ce qui n'est pas absolument nécessaire) ${ }^{101}$. Pour Pausanias, la Sibylle la plus ancienne était la fille de Zeus et de Lamie et sa présence à Delphes est expliquée en faisant de cette femme une prophétesse errante, du nom d'Hérophile (ou même Artémis), qui aurait prophétisé à Samos, Claros, et Délos avant son arrivée à Delphes, et dont se disputaient l'origine Marpessos, Alexandrie de Troade et Érythrées ${ }^{102}$.

Une autre tradition nous parvient à travers d'autres sources. Dans le poème des Épigones (attribué à Antimaque de Téos, vir siècle av. J. C.), on

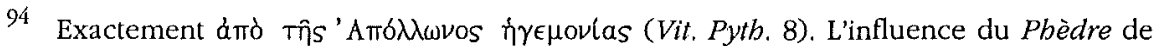
Platon est évidente : $c f$. Giangiulio (2000), II, p. 529. Ce motif de la naissance de Pythagore est également construit sur le modèle de la biographie de Platon : $c f$. les références dans Ramos JuRado (1991), p. 20-21 avec les notes.

95 Vita Pyth., 16.

96 Dans le même chapitre, Porphyre raconte le voyage de Pythagore en Crète où il pratiqua les rites des Dactyles; le philosophe aurait composé également une épigramme pour le tombeau de Zeus. On aperçoit l'intérêt des pythagoriciens pour les histoires de mort (et résurrection) des dieux, ce qui leur permet de les employer pour la doctrine de la métempsychose.

97 Iambl., Vita Pyth., 30; dans le chapitre 135 il affirme qu'Abaris le considérait aussi

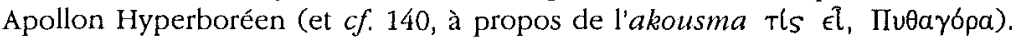

98 Cf. déjà chez Aristote (fr. 191), cité par Élien, V.H. II, 26 (cf. Diog. L., Vita 11).

99 Plut., De Pyth. Or., 9 (398c-e).

100 Cf. Suárez de la TORRe (1994).

101 Strom. I, 15, 70,1.

102 Paus., $\mathrm{X}, 12$. 
racontait comment, après la chute de Thèbes, Manto avait été envoyée comme butin à Delphes, d'où elle était partie (suivant un oracle) afin d'épouser Racios et de s'établir à Colophon (où il fondera le sanctuaire de Claros). Leur fils sera le devin Mopsos ${ }^{103}$. Enfin, Diodore de Sicile ${ }^{104}$ dit que la Sibylle Delphique est en réalité Daphné, la fille de Tirésias. C'est une version intéressante, car elle ferme un cercle mythique assez cohérent : la Daphné du mythe de la métamorphose en laurier (étiologique également) était, d'après certaines versions, fille du fleuve thessalien Péneios, c'est-à-dire qu'elle provient du territoire où Apollon se purifie ${ }^{105}$. L'assimilation à la Sibylle dénote l'influence de la mythologie de Cassandre (causée par la nature des rapports avec Apollon), qui, à son tour, deviendra aussi une "Sibylle » dans la littérature tardive. Signalons que la ville de Tricca frappera des monnaies avec une image d'une Sibylle identifiée comme Manto/ Daphné ${ }^{106}$.

Le facteur commun à ces récits est sans doute la neutralisation d'une mantique en concurrence avec celle de Delphes par l'absorption de la figure de la Sibylle, de la même façon que la légende fait dériver de Delphes la fondation de Claros. En accord avec les légendes qui concernent les poètes et les Pythies, cette inclusion de la Sibylle dans l'entourage delphique confirme une tendance à resserrer les liens entre la poésie sous toutes ses formes (en l'occurrence, en hexamètres notamment) et le sanctuaire apollinien ${ }^{107}$.

Emilio SUÁREZ DE LA TORRE

Universidad de Valladolid

Plaza del Campus, s/n

$\mathrm{E}-470011$ VALLADOLID

emilio@fyl.uva.es

\section{Bibliographie}

AMANDRY, P., La mantique apollinienne à Delphes. Essai sur le fonctionnement de l'Oracle, Paris, 1950.

_, "La vie religieuse à Delphes : bilan d'un siècle de fouilles", in JACQUEmIN (2000), p. 921.

Barrigón Fuentes, Ma.C., "Sobre el culto de las Musas en Delfos", CFC (egi) 6 (1996), p. $237-250$.

Bommelaer, J.-F., Delphes. Centenaire de la "Grande fouille" réalisée par l'École Française d'Athènes (1892-1903), Actes du Colloque Paul Perdrizet, Strasbourg, 6-9 novembre 1991, Leiden, E. J. Brill, 1992.

Bonnechere, P., "La personnalité mythologique de Trophonios", RHR 216 (1999), p. 259297.

${ }^{103}$ Fr. 3 Bernabé, Autant le Pseudo-Apollodore que Pausanias y font référence.

104 IV, 66.

105 Cf. supra.

106 Cf. M. Caccamo Caltabiano, s.v. "Sibyllae" in LIMC VII 1 (1994), p. 753-757.

107 Cf. Maurizio (1998) pour une proposition de rapport entre biographie de la Sibylle, pratiques rituelles et traditions oraculaires. 
Buxton, R. (éd.), From Myth to Reason? Studies in the Development of Greek Thought, Oxford, 1999 .

Chirassi-Colombo, I., "Heros Achilleus - Theos Apollon", in B. Gentili, G. Paione (éds.), Il mito greco. Atti del Convegno Internazionale, Urbino, 7-12 Maggio 1973, Roma, 1977, p. 231-269.

Daux, G., Pausanias à Delphes, Paris, 1936.

Defradas, J., Les thèmes de la propagande delphique, Paris, $1972^{2}$ (1954).

Detienne, M., "Expérimenter dans le champ des polythéismes", Kernos 10 (1997), p. 5772.

-, Apollon le couteau à la main, Paris, Gallimard, 1998.

Dietrich, B,C., "Divine Madness and conflict at Delphi", Kernos 5 (1992), p. 41-58.

Farnell, L.R., The Cults of the Greek States, vol. IV, New York, $1977^{2}$.

Fernández Delgado, J.A., "Das Orakel in der frühgriechischen Poesie", WbJ 17 (1991), p. 17-39.

Fontenrose, J., Python. A Study of Delphic Myth and Its Origins, Berkeley/Los Angeles, University of California Press, 1959.

-, "The Cult and Myth of Pyrrhos at Delphi", in UCPA 4,3 (1960), p. 191-266.

Försted, K. "Zu Pindars achtem Paian", RhM 115 (1972), p. 97-133.

Gadamer, H.G., "Mythos und Vernunft", in Gesammelte Werke, vol VIII, Ästbetik und Poetik, I, Kunst als Aussage, Tübingen, 1993.

Giangiulio, M., Pitagora. Le opere e le testimonianze, I-II, Milan, Mondadori, 2000

JACQUemin, A., Offrandes Monumentales à Delphes, Paris, De Boccard, 1999 (Bibliothèque des Écoles Françaises d'Athènes et de Rome, Fasc. 304).

- (éd.), Delphes cent ans après la Grande fouille. Essai de bilan (Actes du Colloque International organisé par l'École Française d'Athènes, Athènes-Delphes, 17-20 septembre 1992), Paris, De Boccard, 2000 (BCH Suppl. 36).

Kol., D. Der pythische Apollonhymnus als aitiologische Dichtung, Meisenheim am Glan, 1963.

Kritzas, Ch., "Boupyga-Cassotis", BCH 110 (1986), p. 611-617.

Lacrorx, L., "Pausanias et les origines mythiques de Delphes", Kernos 4 (1991), p. 265-276.

Lamprinoudakis, B.K., "Ol $\Delta \in \lambda \phi o l$ kal o ATó $\lambda \lambda \omega \nu$ ", in JACQUemin (2000), p. 23-33.

Loraux, N. Les enfants d'Athéna. Idées athéniennes sur la citoyenneté et la division des sexes, Paris, Seuil, $1990^{2}$.

Malkin, I., "La fondation d'une colonie apollinienne: Delphes et l'Hymne bomérique à Apollon", in JACQUEMIN (2000), p. 69-77.

Maurizio, L., "Narrative, Biographical and Ritual Conventions at Delphi", in I. ChirassiColombo, T. Seppilli, Sibille e linguaggi oracolari. Mito, storia, tradizione, Atti del Convegno Macerata-Norcia, settembre 1994, Macerata, 1998, p. 133-158.

MeyеrноғF, D., Traditioneller Stoff und individuelle Gestaltung. Untersucbungen zu Alkaios und Sappho, Hildesheim/Zürich/New York, 1984.

Мотте, A., "Le premier pèlerinage delphique", in Serta Leodiensia Secunda, Liège, 1992, p. $367-376$.

NAGY, G., The Best of the Achaeans. Concepts of the Hero in Archaic Greek Poetry, Baltimore, 1979 .

OWEN, A.S., Euripides Ion, Oxford, 1939.

PARKE, H.W., "Apollo and the Muses, or Prophecy in Greek Verse", Hermathena 130-131 (1981), p. 99-112.

Parker, R., Miasma. Pollution and Purification in Early Greek Religion, Oxford 1983.

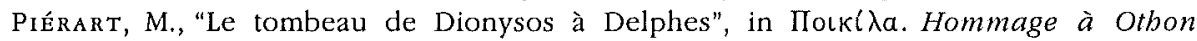
Scholer, Luxembourg, Centre Universitaire, 1996 (Études classiques VIII), p. 137-148. 
Pirenne-Delforge, V., Suárez de la Torre, E. (éds.), Héros et béroïnes dans les mythes et les cultes grecs, Liège, CIERGA, 2000 (Kemos, suppl. 10).

pòrtulas, J., "Poetas míticos de Grecia", in E. Crespo, $\mathrm{M}^{2}$ José Barrios (éds.), Actas del X Congreso Español de Estudios Cläsicos (21-25 de septiembre de 1999), vol. I, Madrid, 2000, p. 289-312.

Quantin, F., "Gaia oraculaire : tradition et réalités", Mètis 7 (1992), p. 177-199.

Ramos Jurado, E., Jámblico. Vida Pitagórica, Madrid, Etnos, 1991.

Rosenberger, V., Griechische Orakel. Eine Kulturgeschichte, Darmstadt, WB, 2001.

Rougemont, G., Corpus des inscriptions de Delphes, I. Lois sacrées et règlements religieux, Paris, De Boccard, 1977.

-, "Énigme à Delphes", in Architecture et poésie. Hommage à G. Roux, Lyon, 1989, p. 225-229.

Roux, G., Delphes. Son oracle et ses dieux, Paris, 1976.

Ruiz Pérez, A., Oráculo y profecía en el mito griego: las familias de Tántalo y Cadmo, Thèse de Doctorat, Université de Valladolid, 1995.

Rutherford, I., Pindar's Paeans. A Reading of the Fragments with a Survey of the Genre, Oxford, 2001.

SánCHez, P., L'Ampbictionie des Pyles et de Delphes. Recberches sur son rôle bistorique des origines au II siècle de notre ère, Stuttgart, Franz Steiner, 2001.

Sourvinou-Inwoon, C., "The Myth of the Four Temples at Delphi", CQ 29 (1979), p. 231251 (= Reading Greek Culture, Oxford 1991, p. 192-216).

-, "Myth as History: The Previous Owners of the Delphic Oracle", in J. Bremmer (éd.), Interpretations of Greek Mythology, London 1987, p. 215-241 (= Reading Greek Culture, Oxford, 1991, p. 217-243).

Stafford, E., Worshipping Virtues. Personification and the Divine in Ancient Greece, London, Duckworth, 2000.

Suárez de la TORRE, E., "Sibylles, mantique inspirée et collections oraculaires", Kernos 7 (1994), p. 179-205.

—, "Neoptolemos at Delphi", Kernos 10 (1997), p. 153-176.

-, "Cuando los límites se desdibujan: Dioniso y Apolo en Delfos", in C. SÁnchez

Fernández, P. Cabrera Bonet, En los limites de Dioniso (Actas del Simposio celebrado en el Museo Arqueológico Nacional, Madrid, 20 de junio de 1997), Murcia, 1998 (a), p. $17-28$.

-, "Algunos motivos de la mitología apolínea y su trasfondo religioso", in J.L. Calvo Martínez (éd.), Religión, magia y mitología en la Antigïedad clásica, Granada, 1998 (b), p. 283-330 (Biblioteca de Estudios Clásicos, Universidad de Granada).

-, "Observaciones sobre los rituales délficos eneaetéricos", in Corolla Complutensis (Homenaje al Profesor José S. Lasso de la Vega), Madrid, 1998 (c), p. 469-482.

—, "Les dieux de Delphes et l'histoire du sanctuaire (des origines au iv e siècle av. J. C.)", in V. Pirenne-Delforge (éd.), Les Panthéons des cités des origines à la Périégèse de Pausanias, Liège, CIERGA, 1998 (d), p. 61-89.

Usener, H., "Heilige Handlung", $A R W 7$ (1904), p. 281-339 (= Kleine Schriften IV, Leipzig/Berlin, 1913, p. 422-467).

Watkins, C., How to kill a Dragon. Aspects of Indoeuropean Poetics, New York/Oxford, 1995.

Woodbury, L., "Neoptolemos at Delphi: Pindar Nem. 7, 30 ff.", Pboenix 33 (1979), p. 95133. 


\section{Appendice : Cadre des généalogies mythiques de Delphes}

$$
\text { (Paus., X, 6, } 1 \text { sq. + VII, 18, 9) }
$$

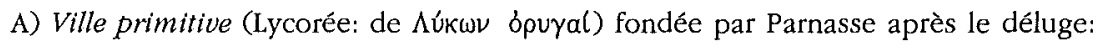

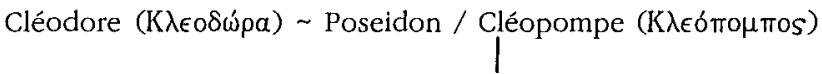

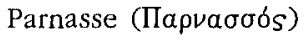

B) Versions alternatives (et généalogies diverses)

1. Apollon Nymphe Coryce (Kwpukla)

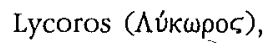

2. Lycoros

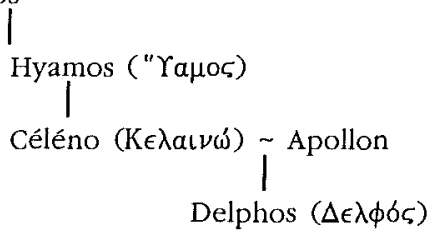

3. Achéloos / Céphise Castalie (Thyie?) [cf. Hdt., VII, 178]

4. Céphise (Kєфıớs)

I Mélène (Métiaıva) Apollon<smiles>[TlH]</smiles>

Delphos

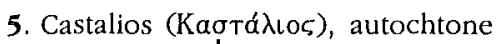

$$
\text { I }
$$

Thyie $(Q u i \alpha=$ Castalie? $) \sim$ Apollon

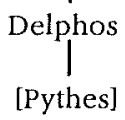

6. Delphos

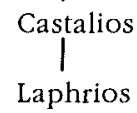

7. [Douteux, vid. $C I D$ 9, D 31-33]

Cas(s)otos (Kav(o)otós)

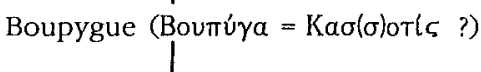

Labys ( $\Lambda a ́ \beta u \varsigma$ - $\Lambda a \beta v a ́ \delta a \iota)$
8. Schol. E. Or, 1094 :

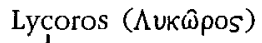

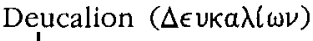

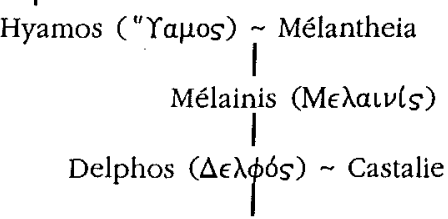

Castalios Phémonoé

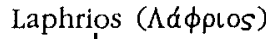
I Noutios (Noútios) 